\title{
Microsurgical anatomy of the subthalamic nucleus: correlating fiber dissection results with 3-T magnetic resonance imaging using neuronavigation
}

\author{
Abuzer Güngör, MD, $, 1,2$ Şevki Serhat Baydın, MD, ${ }^{3}$ Vanessa M. Holanda, MD, PhD, ${ }^{4}$ \\ Erik H. Middlebrooks, MD, ${ }^{5}$ Cihan Isler, MD, ${ }^{6}$ Bekir Tugcu, MD, ${ }^{2}$ Kelly Foote, MD, ${ }^{4}$ and \\ Necmettin Tanriover, MD $^{6}$
}

\begin{abstract}
${ }^{1}$ Department of Neurosurgery, Acıbadem University; ${ }^{2}$ Department of Neurosurgery, Bakirkoy Research \& Training Hospital for Psychiatry, Neurology, and Neurosurgery; ${ }^{3}$ Department of Neurosurgery, Kanuni Sultan Süleyman Research \& Training Hospital; ${ }^{6}$ Department of Neurosurgery, Cerrahpasa Medical Faculty, Istanbul University, Istanbul, Turkey; ${ }^{4}$ Department of Neurosurgery, University of Florida, Gainesville, Florida; and ${ }^{5}$ Department of Radiology, University of Alabama, Birmingham, Alabama
\end{abstract}

\begin{abstract}
OBJECTIVE Despite the extensive use of the subthalamic nucleus (STN) as a deep brain stimulation (DBS) target, unveiling the extensive functional connectivity of the nucleus, relating its structural connectivity to the stimulation-induced adverse effects, and thus optimizing the STN targeting still remain challenging. Mastering the 3D anatomy of the STN region should be the fundamental goal to achieve ideal surgical results, due to the deep-seated and obscure position of the nucleus, variable shape and relatively small size, oblique orientation, and extensive structural connectivity. In the present study, the authors aimed to delineate the 3D anatomy of the STN and unveil the complex relationship between the anatomical structures within the STN region using fiber dissection technique, 3D reconstructions of high-resolution $\mathrm{MRI}$, and fiber tracking using diffusion tractography utilizing a generalized q-sampling imaging (GQI) model.
\end{abstract}

METHODS Fiber dissection was performed in 20 hemispheres and 3 cadaveric heads using the Klingler method. Fiber dissections of the brain were performed from all orientations in a stepwise manner to reveal the $3 \mathrm{D}$ anatomy of the STN. In addition, 3 brains were cut into 5-mm coronal, axial, and sagittal slices to show the sectional anatomy. GQI data were also used to elucidate the connections among hubs within the STN region.

RESULTS The study correlated the results of STN fiber dissection with those of 3D MRI reconstruction and tractography using neuronavigation. A 3D terrain model of the subthalamic area encircling the STN was built to clarify its anatomical relations with the putamen, globus pallidus internus, globus pallidus externus, internal capsule, caudate nucleus laterally, substantia nigra inferiorly, zona incerta superiorly, and red nucleus medially. The authors also describe the relationship of the medial lemniscus, oculomotor nerve fibers, and the medial forebrain bundle with the STN using tractography with a 3D STN model.

CONCLUSIONS This study examines the complex 3D anatomy of the STN and peri-subthalamic area. In comparison with previous clinical data on STN targeting, the results of this study promise further understanding of the structural connections of the STN, the exact location of the fiber compositions within the region, and clinical applications such as stimulation-induced adverse effects during DBS targeting.

https://thejns.org/doi/abs/10.3171/2017.10.JNS171513

KEYWORDS subthalamic nucleus; Parkinson's disease; globus pallidus internus; neuroanatomy; functional neurosurgery; anatomy; zona incerta; deep brain stimulation

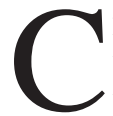
HRONIC deep brain stimulation (DBS) of the subthalamic nucleus (STN) is a proven neurosurgical treatment to relieve refractory motor symptoms in Parkinson's disease. ${ }^{6,9,26,32,41,58-61}$ Preliminary results also suggest that STN stimulation may also be beneficial in cases of refractory obsessive-compulsive disorder and epilepsy. ${ }^{11,13,15,28,56,60,61}$ Despite the extensive use of the STN as a DBS target, understanding of the 3D anatomy of the subthalamic region remains challenging due to the variable shape, oblique orientation, relatively small size, and

ABBREVIATIONS DBS = deep brain stimulation; GPe = globus pallidus externus; $\mathrm{GPi}=$ globus pallidus internus; $\mathrm{GQI}=$ generalized q-sampling imaging; IC = internal capsule; LGB = lateral geniculate body; MFB = medial forebrain bundle; SLF = superior longitudinal fasciculus; STN = subthalamic nucleus; ZI = zona incerta.

SUBMITTED June 22, 2017. ACCEPTED October 18, 2017.

INCLUDE WHEN CITING Published online May 4, 2018; DOI: 10.3171/2017.10.JNS171513. 
extensive connectivity of the nucleus. The STN's intimate relationships with other elusive deep brain structures, such as the zona incerta (ZI), ansa lenticularis, medial forebrain bundle (MFB), medial lemniscus, and substantia nigra, further challenge precise targeting in STN-DBS lead implantation. . $2,22,37,43,50,57,64^{2}$

3D computer reconstruction of the STN has been shown to be effective for preoperative target identification before DBS surgery as well as for intraoperative recogni-

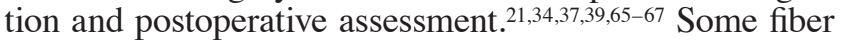
dissection studies have also been performed to describe STN anatomy. 1,12,64 However, no study in the literature has combined 3D MRI reconstruction with generalized qsampling imaging (GQI) tractography and sectional anatomy of the STN through the fiber dissection technique. We aimed to elucidate the 3D anatomy of the STN and related structures using the fiber dissection technique, 3D reconstruction of high-resolution MRI, and GQI tractography.

\section{Methods}

Ten brains (20 hemispheres) and 3 heads were obtained from the State of Florida Anatomical Board and fixed with $10 \%$ formalin for at least 1 month. All specimens were verified to lack a central nervous system pathology that would skew the findings. After fixation, all specimens were frozen in water for at least 2 weeks at $-16^{\circ} \mathrm{C}$ to facilitate dissection, in accordance with the Klingler method..$^{18,29}$ The arteries and veins of the whole heads were injected with blue or red silicone rubber (Dow Corning Corp.), RTV catalyst (Dow Corning Corp.), and Thinner 200 (Dow Corning Corp.). The dura mater, arachnoid membrane, and surface vessels were removed carefully under the microscope (Carl Zeiss AG) with $6 x-40 x$ magnification after at least 1 hour of thawing under tap water. The dissections were performed with Rhoton dissectors. Between dissections, the specimens were kept in a 70\% alcohol solution at room temperature. The dissections were performed in a stepwise manner from lateral to medial, medial to lateral, superior to inferior, and inferior to superior to reveal the 3D anatomy of the STN. Before the cranial dissections were started, 3-T MRI with 1-mm slices was acquired in each head specimen for MR navigation (Medtronic StealthStation S7). In addition, 3 brains were cut into 5-mm coronal, axial, and sagittal slices to show the sectional anatomy. Each stage of the dissections was recorded with digital photography.

Nuclei were manually labeled on a voxel-by-voxel basis in DSI Studio (http://dsi-studio.labsolver.org). T1 MPRAGE (magnetization-prepared rapid gradient echo) MRI data were acquired on a modified 3-T Siemens Skyra (Siemens AG) with parameters as follows: $0.7 \times 0.7 \times 0.7-\mathrm{mm}$ voxel size, TR $2400 \mathrm{msec}$, TE $2.14 \mathrm{msec}$, TI $1000 \mathrm{msec}$, flip angle $8^{\circ}$, and GRAPPA 2. The MP-RAGE data were registered to a multidirection diffusion dataset, which was processed using a generalized q-sampling method as described in previous publications. ${ }^{4}$ Voxels were validated as gray matter by defining those voxels with a quantitative anisotropy (QA) threshold above 0.13 to allow delineation of nuclei borders. Fiber tracking was performed by deter- ministic tractography using seed points placed along the expected course of the white matter tracts of interest. A termination index of $\mathrm{QA}=0.08$, angle $>75^{\circ}$, a step size of $0.67 \mathrm{~mm}$, and a smoothing of $0.2 \mathrm{~mm}$ were used.

\section{Results \\ Fiber Dissection En Route to the STN}

Removing the cortex from the lateral surface of the hemisphere, the superior longitudinal fasciculus (SLF) II and III, and the arcuate fasciculus exposes the corona radiata anteriorly, the sagittal stratum posteriorly, and the insular cortex centrally (Fig. 1A and B). Removing, one by one, the insular cortex, the extreme capsule, and the deeper external capsule layers, which contain claustrocortical fibers dorsally and inferior fronto-occipital and uncinate fibers ventrally, displays the putamen (Fig. 1CE). Removing the putamen exposes the globus pallidus, anterior commissure, and internal capsule (Figs. $1 \mathrm{~F}$ and $2 \mathrm{~A}-\mathrm{F}$ ). The putamen and globus pallidus form a cone-like shape, with its floor corresponding to the lateral surface of the putamen and its apex to the globus pallidus internus (GPi), which points toward the peri-STN region and substantia nigra. The GPi is located superior to the optic tract. The fibers arising from GPi and curving caudally to pass ventral to the IC along the way to the STN constitute the ansa lenticularis, which is located between the GPi and the optic tract (Fig. 2A-F).

The internal capsule (IC) is a heterogeneous structure that consists of fibers coursing along the neuraxis, some of which reach the brainstem. At the upper edge of the putamen along the superior limiting sulcus of the insula, the IC joins with fibers from the external capsule to form the corona radiata. The fiber system of the IC consists of the anterior limb, genu, posterior limb, and the retro- and sublenticular parts. The anterior limb of the IC forms a border between the putamen and caudate nucleus. The posterior limb separates the globus pallidus from the thalamus and STN (Figs. 1F, 2A-E, 3E and F, 4C and D, and 5A and $\mathrm{B})$. The anterior limb includes the anterior thalamic radiations and frontopontine tract, which arise from the frontal lobe cortex. The genu includes the anterior part of the corticobulbar and corticospinal tracts and the anterior part of the superior thalamic peduncle. The posterior limb includes the posterior part of the corticospinal tract and the parietal thalamic peduncle and parietopontine fibers. The retrolenticular part of the IC includes the parietopontine fibers and part of the optic radiation, and the sublenticular part contains occipitopontine and temporopontine fibers, as well as the anterior component of the optic radiation (Figs. 1F, 2A-E, 3E and F, and 5A-D).

Removing the IC fibers exposes the thalamus, caudate nucleus, and amygdala (Figs. 2C, D, and F, 3B and F, and $4 \mathrm{~A}$ and $\mathrm{B}$ ). The $\mathrm{C}$-shaped caudate nucleus wraps around the thalamus and can be divided into 3 parts: the head, body, and tail (Fig. 2C and D). The head of the caudate nucleus is located at the lateral wall of the frontal horn of the lateral ventricle, the body at the lateral wall of the body of the lateral ventricle, and the tail at the roof of the temporal horn of the lateral ventricle. The thalamus is located at the internal edge of the caudate nucleus (Figs. 2C and 

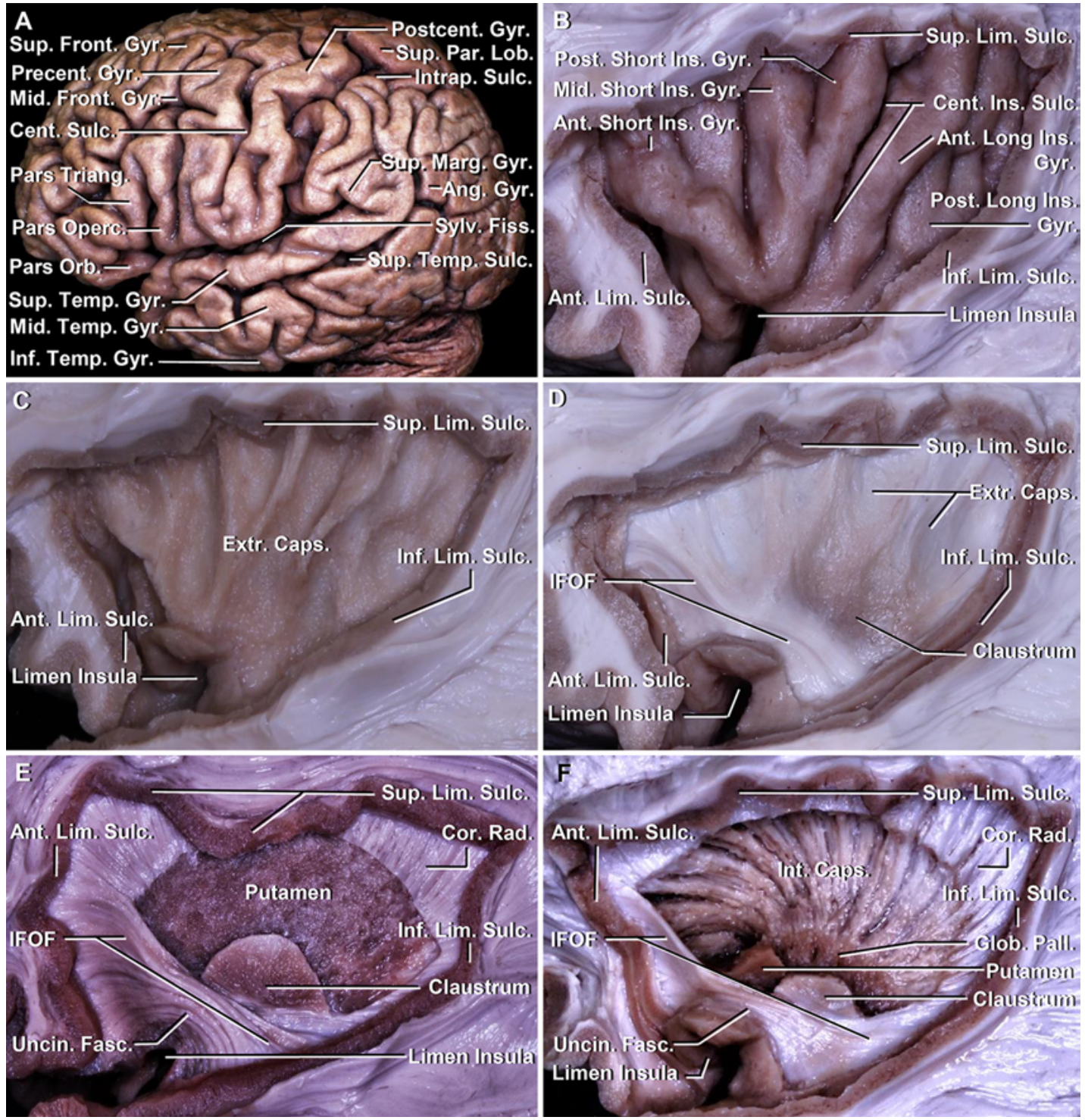

FIG. 1. Lateral to medial fiber dissection of a left hemisphere to display the relations of the subthalamic region in a stepwise systematic manner. A: Identification of sulci and gyri on the lateral surface of the left hemisphere. B: The pyramid-shaped insula has been exposed by removing the cortical surface and underlying white matter along the frontoparietal and temporal operculi. $\mathbf{C}$ and D: Initially, the extreme capsule has been displayed through a decortication process along the insular cortex (C). The external capsule layer has been reached following the removal of the extreme capsule (D). Deeper external capsule layer contains dorsally located claustrocortical fibers and ventrally located inferior fronto-occipital and uncinate fasciculi. $\mathrm{E}$ and $\mathrm{F}$ : The putamen and globus pallidus, in addition to the $\mathrm{IC}$, have been revealed by continuing the lateral to medial fiber dissection. Removing the ventral external capsule fibers exposed the putamen and, by removing this structure, the IC fibers and globus pallidus have been displayed. Ang. = angular; Ant. = anterior; Caps. = capsule; Cent. = central; Cor. = corona; Extr. = extreme; Fasc. = fasciculus; Fiss. = fissure; Front. = frontal; Glob. = globus; Gyr. = gyrus; IFOF = inferior fronto-occipital fasciculus; Inf. = inferior; Ins. = insular; Int. = interna, internal; Intrap. = intraparietal; Lim. = limiting; Lob. = lobule; Marg. = marginal; Mid. = middle; Operc. = opercularis; Orb. = orbitalis; Pall. $=$ pallidus; Par. $=$ parietal; Post. $=$ posterior; Postcent. $=$ postcentral; Precent. $=$ precentral $;$ Rad.$=$ radiata, radiation; Sulc. $=$ sulcus; Sup. = superior; Sylv. = sylvian; Temp. = temporal; Triang. = triangularis; Uncin. = uncinate. Copyright Necmettin Tanriover. Published with permission. Figure is available in color online only.

$\mathrm{D}, 3 \mathrm{~B}$, and $4 \mathrm{~A}$ and $4 \mathrm{~B})$. The anterior part of the thalamus is located at the floor of the body of the lateral ventricle, and the posterior part of the thalamus is located at the anterior wall of the atrium and superomedial wall of the temporal horn. The lateral geniculate body (LGB), located posteroinferiorly at the lateral part of the thalamus, is the point at which the optic tract ends and the optic radiation begins. The stria terminalis, another $\mathrm{C}$-shaped bundle, lies between the thalamus and the caudate nucleus (Figs. 2F, $4 \mathrm{D}$, and $5 \mathrm{~A}$ and $\mathrm{B}$ ). The amygdala is located at the anterior wall of the temporal horn just anterior to the hippocampus and lateral to the inferolateral part of the thalamus. It is continuous with the globus pallidus superomedially and the caudate tail posteromedially (Fig. 2F). 

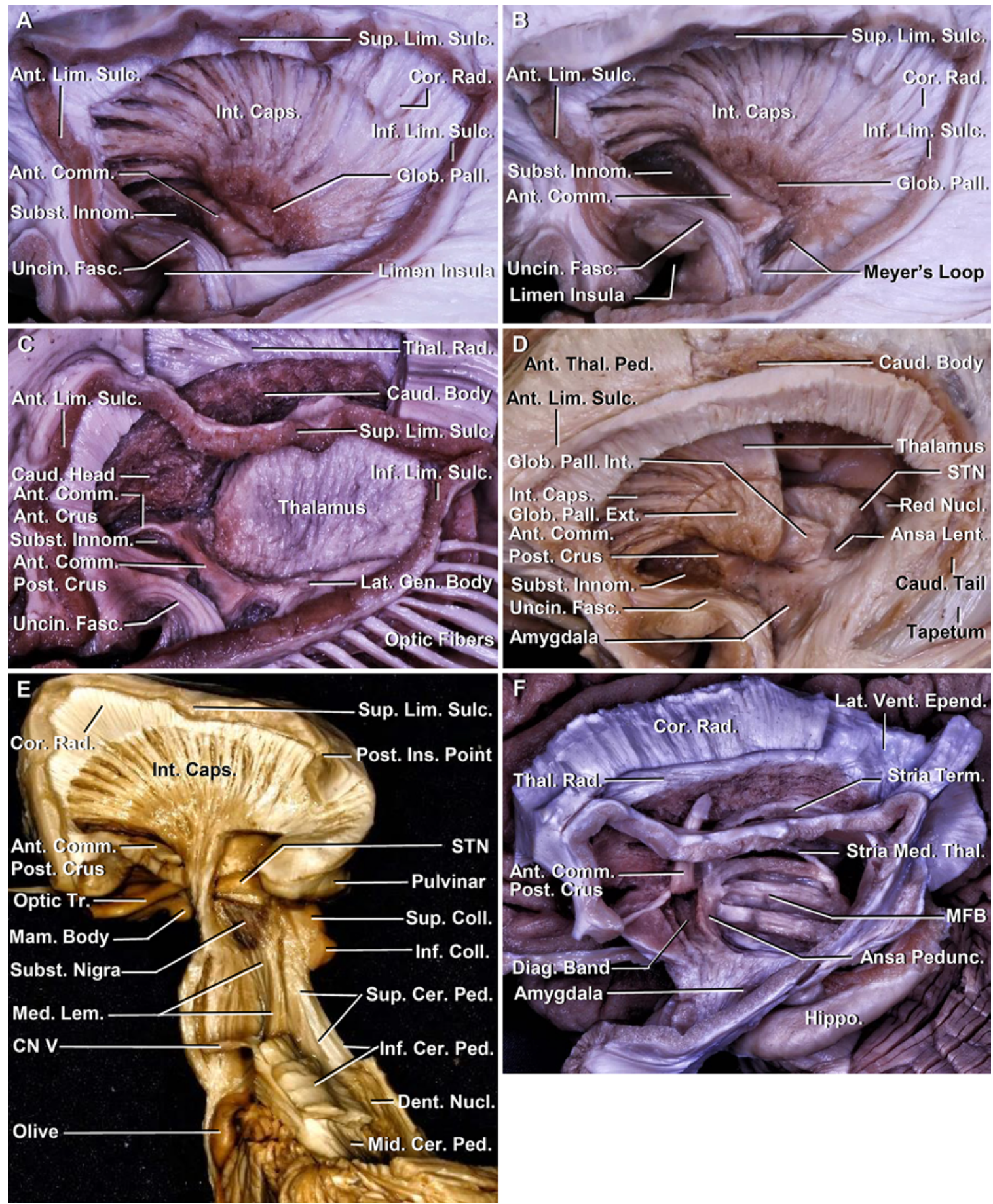

FIG. 2. A: Further lateral to medial dissection. The anterior commissure has been exposed through further medial fiber dissection. The anterior commissure, passing just inferior to the middle base of the globus pallidus, has been shown, along with its temporal and occipital extensions. The division along the temporal stem is located near the temporal edge of the limen and proximal to the inferior limiting sulcus of insula. B: Retrolenticular and sublenticular parts of the IC, including the anterior part of the optic radiations, have been shown deep to the anterior commissure layer. Meyer's loop consists of fibers arising from the lateral geniculate body, running anteriorly with a posterior curve at the temporal pole. C: Caudate nucleus medial to IC and thalamic radiations has been demonstrated by moving medially to the next step through the fiber dissection on the same hemisphere. C-shaped caudate nucleus, circling the anterosuperior and superior boundaries of the thalamus, has been exposed by removing the IC. D: The STN laterally, red nucleus inferomedially, and substantia nigra inferiorly have been identified by removing the posterior part of the thalamus in another left hemisphere. The ansa lenticularis, composed of pallidofugal fibers, lies between the globus pallidus and STN within the prerubral region. The STN, with its almond-like shape, is located superolateral to the red nucleus, medial to the GPi, and superior to the substantia nigra. FIG. 2. (continued) $\rightarrow$ 
FIG. 2. E: The posterior part of the IC and thalamus have been removed to show substantia nigra and STN in relation to brainstem and cerebellum in another left hemisphere. Removing the transverse pontine fibers exposes medial lemniscus and cerebellar peduncles. The medial lemniscus runs from the gracile and cuneate tubercles to the ventral posterolateral nucleus of the thalamus and divides the brainstem into ventral and dorsal parts. The medial lemniscus fibers intermingle with substantia nigra and pass inferomedial to the STN before terminating in the thalamus. Corticospinal, corticobulbar, corticomesencephalic fibers have a close relationship with the lateral anterior and inferior borders of the STN throughout their descending path trajectory toward the pons within the IC. F: The ansa peduncularis and medial forebrain bundle have been exposed by removing the globus pallidus, IC fibers, and thalamus in another specimen on the left side. The medial forebrain bundle connects the lateral hypothalamus and the septal area with the ventral tegmental area. The medial forebrain bundle lies at the level of and just lateral to the red nucleus, lateral to the mammillothalamic tract, medial to the substantia nigra, and slightly inferomedial to the STN. Caud. = caudate; Cer. = cerebellar; $\mathrm{CN}$ = cranial nerve; Coll. = colliculus; Comm. = commissure; Diag. = diagonal; Epend. = ependyma; Ext. = externa; Gen. = geniculate; Hippo. = hippocampus; Innom. = innominata; Lat. = lateral; Lem. = lemniscus; Lent. = lenticularis; Mam. = mammillary; Med. = medial, medullaris; Nucl. = nucleus; Ped. = peduncle; Pedunc. = peduncularis; Subst. = substantia; Term. = terminalis; Thal. = thalami, thalamic; Tr. = tract; Vent. = ventricle. Copyright Necmettin Tanriover. Published with permission. Figure is available in color online only.
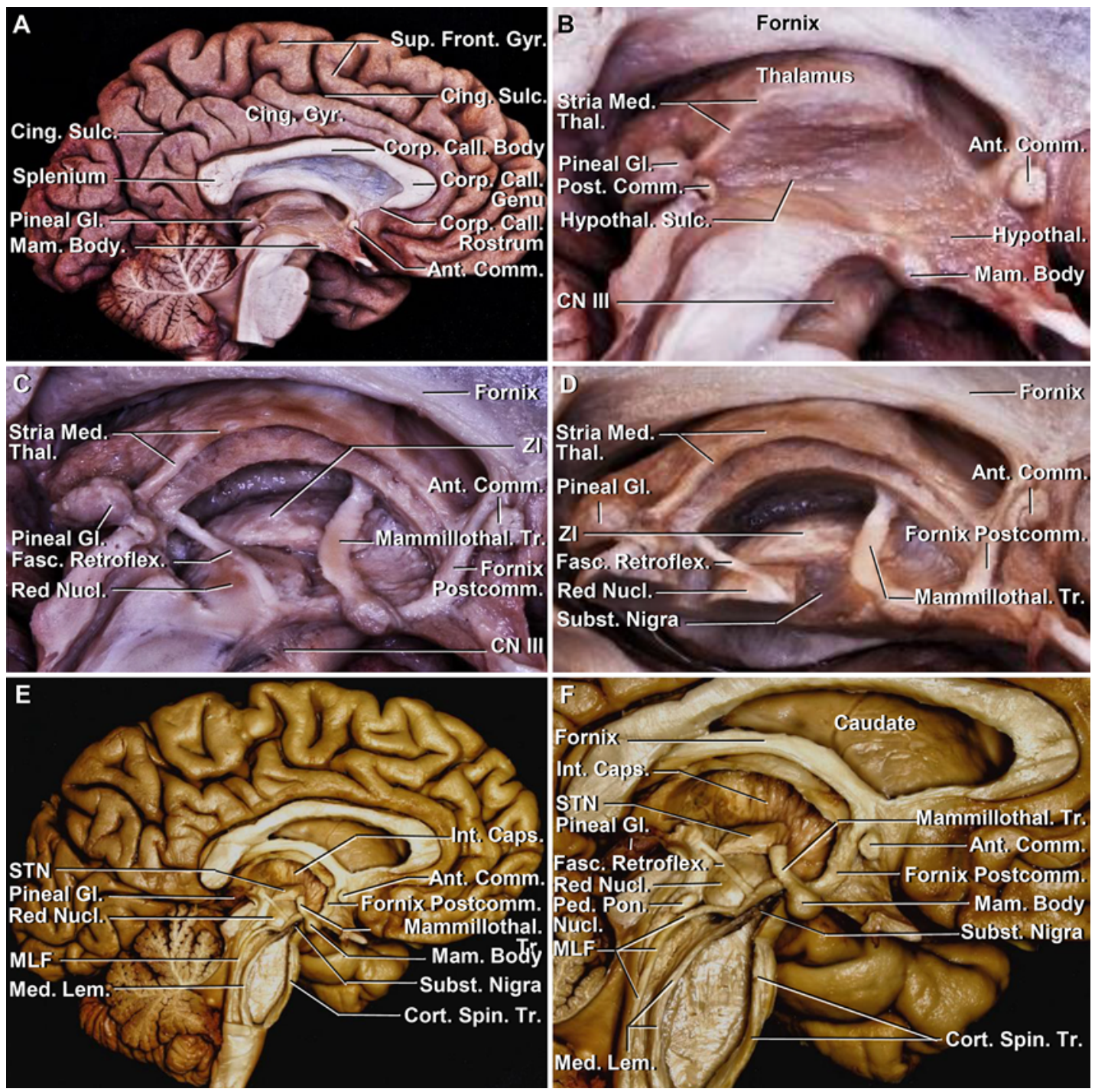

FIG. 3. Medial to lateral fiber dissection of a left hemisphere to display the relations of the subthalamic region in a stepwise manner. A: Identification of sulci and gyri on the medial surface of the left hemisphere. B: Enlarged view of panel A, displaying the 3rd ventricular region. C: The fasciculus retroflexus, red nucleus, Zl, mammillothalamic tract, and postcommissural fornix have been displayed by removing the ventral part of the thalamus through a fiber dissection on the medial side of a left cerebral hemisphere. The fasciculus retroflexus lies between the habenula and interpeduncular nucleus just medial to the red nucleus. The red nucleus is visible medial and inferior to the ZI and posterior to the mammillothalamic tract. The ZI has been exposed under the thalamus and covers the STN on the dorsomedial side. D: The anterior part of the red nucleus has been removed during further medial to lateral dissection. The rostral part of the ZI extends over the dorsal and medial surfaces of the STN and its caudal part lies posteromedial to the nucleus. The substantia nigra spreads from the inferior part of the red nucleus to the inferior border of the ZI and STN. FIG. 3. (continued) $\rightarrow$ 
FIG. 3. E: Fiber dissection of the subthalamic area in another left medial hemisphere. The ZI, superior part of the mammillothalamic tract, and transverse pontine fibers have been removed and the brainstem has been dissected to expose the STN and medial lemniscus. F: Enlarged view of panel E. Following the removal of the ZI, a biconvex-shaped STN has been exposed medial to the IC fibers. The STN is located superolateral to the red nucleus and superior to the substantia nigra. The IC fibers run from its inferior to anterolateral side. The medial lemniscus traverses the thalamus posterior to the corticospinal tract, before terminating in the thalamus. The medial lemniscus ascends posteroinferior to the STN and is located posterior to the nucleus at this level. Call. = callosum; Cing. = cingulate; Corp. = corpus; Cort. = cortico; Gl. = gland; Hypothal. = hypothalamic, hypothalamus; Mammillothal. = mammillothalamic; MLF = medial longitudinal fasciculus; Ped. = pedunculo; Pon. = pontine; Postcomm. = postcommissural; Retroflex. = retroflexus; Spin. = spinal. Copyright Necmettin Tanriover. Published with permission. Figure is available in color online only.
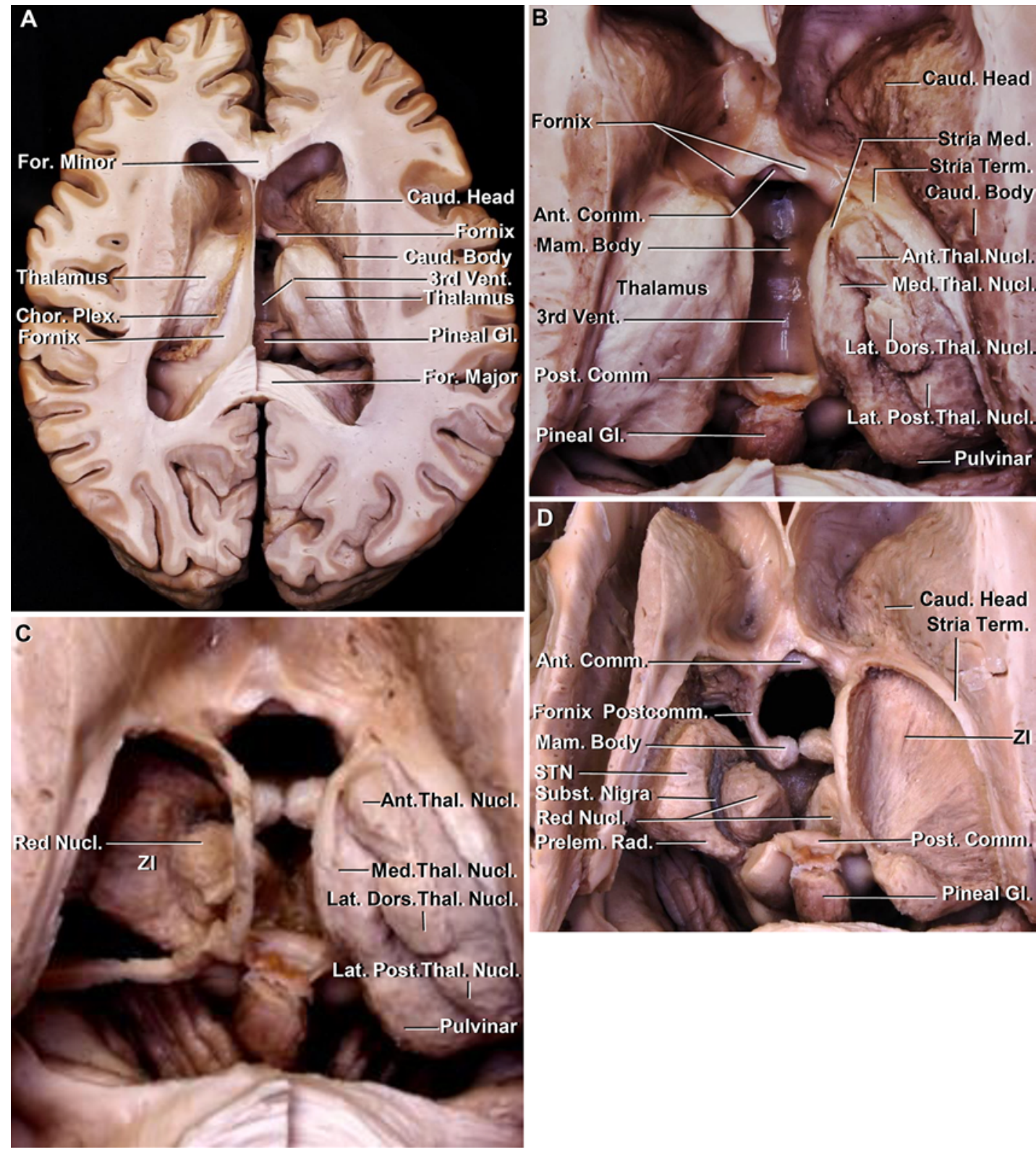

FIG. 4. Superior to inferior fiber dissection of the subthalamic region in a stepwise manner. A: The brain has been cut at the level of the sylvian fissure to demonstrate a superior to inferior fiber dissection toward the subthalamic region. The anterosuperior parts of the caudate nuclei, parts of the fornix, the septum pellucidum, and callosal fibers have been exposed along the frontal horn, body, and atrium of the lateral ventricle. On the right side, the fornix and choroid plexus have been removed to show the anterolateral and posteromedial parts of the thalamus separated by the choroidal fissure. B: The fornix and choroid plexus along the choroidal fissure have been removed via further superoinferior dissection on both hemispheres and the tenia thalami has been removed on the right side. The respective thalamic nuclei, anterior thalamic nucleus, lateral dorsal nucleus, lateral posterior nucleus, medial nuclear group, and pulvinar, in addition to the stria terminalis and stria medullaris thalami, have been exposed. C: The thalamus has been removed on the left side to display the $\mathrm{Zl}$ and the red nucleus through further fiber dissection directed inferiorly. The ZI is located lateral and superior to the red nucleus. The ZI covers the STN dorsally and is located posterosuperior and slightly medial to the STN. FIG. 4. (continued) $\rightarrow$ 
FIG. 4. D: The thalamus has been removed on both sides at this step of dissection. The red nucleus is located just lateral to the midline, posterior to the mammillary body, and the ZI lies between the IC and the red nucleus on the right side. The ZI has been removed on the left side to expose a biconvex-shaped STN, medial to the IC fibers. The STN is located superolateral to the red nucleus. Comparing fiber dissections on both hemispheres in this view places the STN ventral, anteroinferior, and slightly lateral to the Zl. Chor. = choroid; Dors. = dorsal; For. = forceps; Plex. = plexus; Prelem. = prelemniscal. Copyright Necmettin Tanriover. Published with permission. Figure is available in color online only.
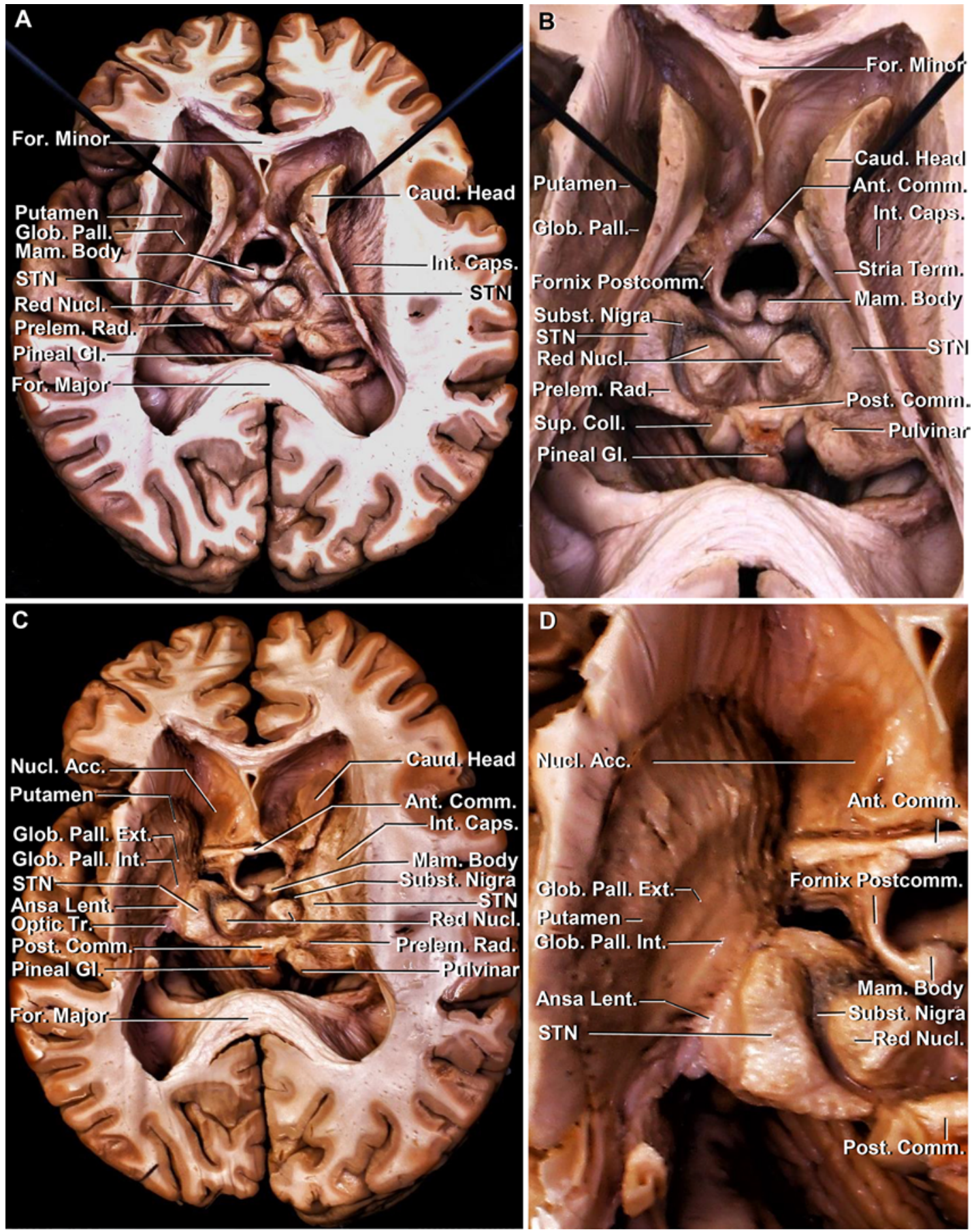

FIG. 5. Further superior to inferior dissection. A: The ZI has been dispatched on the right side and IC fibers have been removed on the left side. B: Enlarged view of Fig. 4E. The head of the caudate nucleus has been reflected superomedially on both sides to expose the IC fibers on the right side and the globus pallidus and putamen on the left side. The STN is located medial to the IC and globus pallidus. The pulvinar of thalamus and prelemniscal radiations are situated posterior to the STN. C: By proceeding with superior to inferior fiber dissection, the caudate nucleus has been removed on the left side, and the head has been preserved on the right. The GPi is located just lateral to the STN and the optic tract lies just under the GPi, inferomedial to the STN. FIG. 5 . (continued) $\rightarrow$ 
FIG. 5. D: Enlarged view of panel A. On the left hemisphere, the nucleus accumbens has been exposed under and extending beneath the head of the caudate nucleus. The substantia nigra spreads under the STN and red nucleus. The ansa lenticularis lies between the GPi and STN. Coll. = collateral, colliculus; Inf. = inferior. Copyright Necmettin Tanriover. Published with permission. Figure is available in color online only.

\section{Subthalamic Area in Relation to the STN}

The area between the thalamus and the hypothalamus is called the subthalamus and includes the zona incerta (ZI) and the STN (Figs. 3A-F, 4C and D, and 5A and B). Removing the thalamus during lateral to medial dissections exposes the ZI, a broad and shallow band of gray matter lying under the thalamus and medial to the IC (Figs. 3C and $\mathrm{D}$ and $4 \mathrm{C}$ and $\mathrm{D})$.

The ZI, a superior continuation of the brainstem reticular formation, is enveloped by efferents from the GPi that pass to the ventrolateral and ventral anterior nuclei of the thalamus. It is difficult to separate the ventral part of the thalamus from the ZI because of their similar external appearance at this stage of dissection, although the ZI emerges as a brighter area of gray matter at higher magnification. Following the removal of the thalamic fasciculus below the ZI, a biconvex-shaped nucleus is exposed medial to the IC fibers. This ovoid nucleus, the STN, is located ventral, anteroinferior, and slightly lateral to the ZI. From a different point of view, the rostral part of the ZI extends over the dorsal and medial surfaces of the STN and its caudal part lies posteromedial to the STN (Figs. 3E and F, $4 \mathrm{D}$, and $5 \mathrm{~A}-\mathrm{D}$ ).

At this point, it can be seen that the most inferior part of the thalamus is situated lower than the level of the STN. Proceeding more medially, the red nucleus, with its spheroid shape, can be defined adjacent to the midline and medial and posteroinferior to the STN. The substantia nigra, with its darker color compared with neighboring structures, spreads along the subthalamic area inferior to the STN and the red nucleus (Fig. 6A and B). The ansa lenticularis, composed of pallidofugal fibers, lies between the GPi and STN within the prerubral region (Figs. 2D and 5C and D). The ansa lenticularis initially travels inferomedially through the posterior limb of the IC toward the STN, passes inferior to the STN, and stays medial to the nucleus during its final ascent (Figs. 2D and 5C and D). The ZI and substantia nigra overlap in the posterior subthalamic area, just posterior to the STN. According to the results of the fiber dissection, the STN is covered by the ZI dorsomedially, the substantia nigra inferiorly, the red nucleus medially, and the IC anterolaterally (Figs. 7A-C, 8A-F, and $9 \mathrm{~A}$ and $\mathrm{B}$ ).

\section{Peri-Subthalamic Area}

Several medial neural structures are intimately related to the STN and may be encountered during surgical approaches to the nucleus. The hypothalamus is located anteromedial to the STN and lies immediately posterior and inferior to the anterior commissure (Fig. 3B). The internal course of the oculomotor nerve and its nucleus are also related to the subthalamic area. The nerve arises from its nucleus lateral to the cerebral aqueduct at the level of the superior colliculi. The fibers from the nucleus of the third cranial nerve, located in the peri-aqueductal gray matter
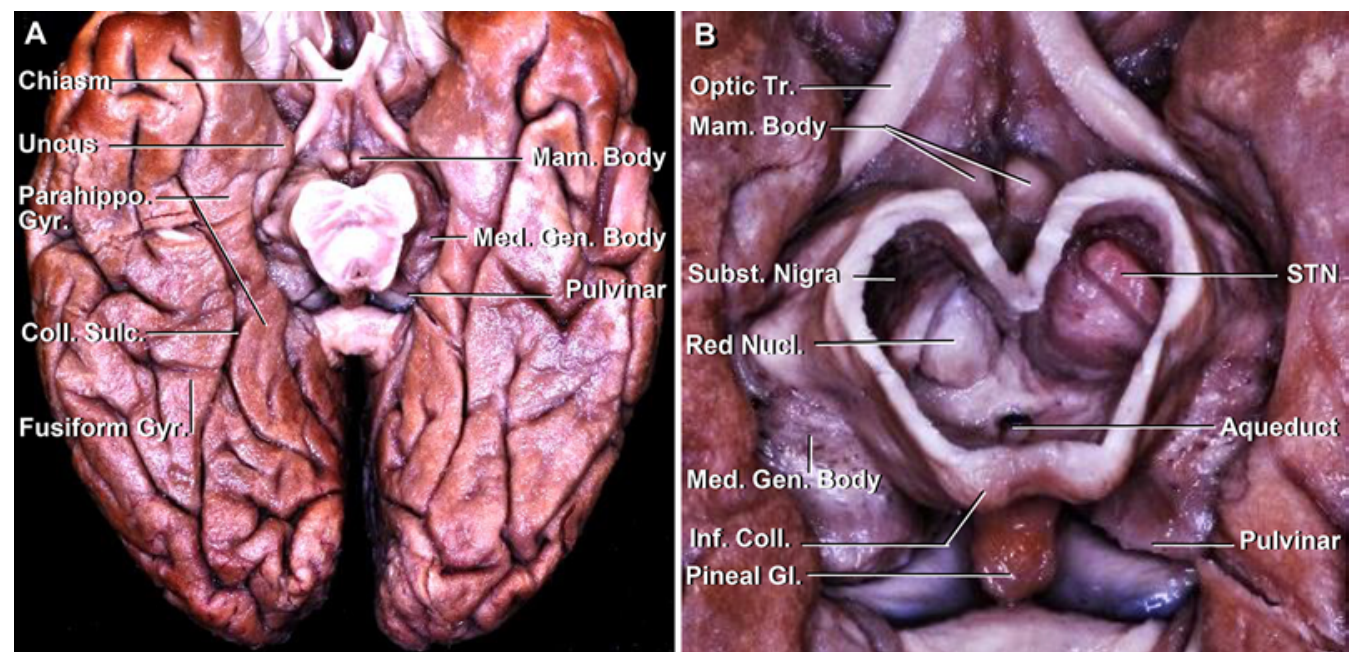

FIG. 6. Inferior to superior fiber dissection of the subthalamic region in a stepwise manner. A: The brainstem has been cut at the level of the inferior colliculi. The medial geniculate body is located at the superior edge of the lateral mesencephalic sulcus, and the optic tract runs from the chiasm to the lateral geniculate body above the uncus. B: Enlarged view of panel A and a further stage on the inferior to superior fiber dissection of the diencephalic region. The substantia nigra, underneath the subthalamic and red nuclei, has been displayed by removing the transverse pontine fibers and the corticobulbar and corticospinal tracts. On the right side, portions of the red nucleus and substantia nigra were removed to better expose the STN with its oblique shape. Parahippo. Gyr. = parahippocampal gyrus. Copyright Necmettin Tanriover. Published with permission. Figure is available in color online only. 

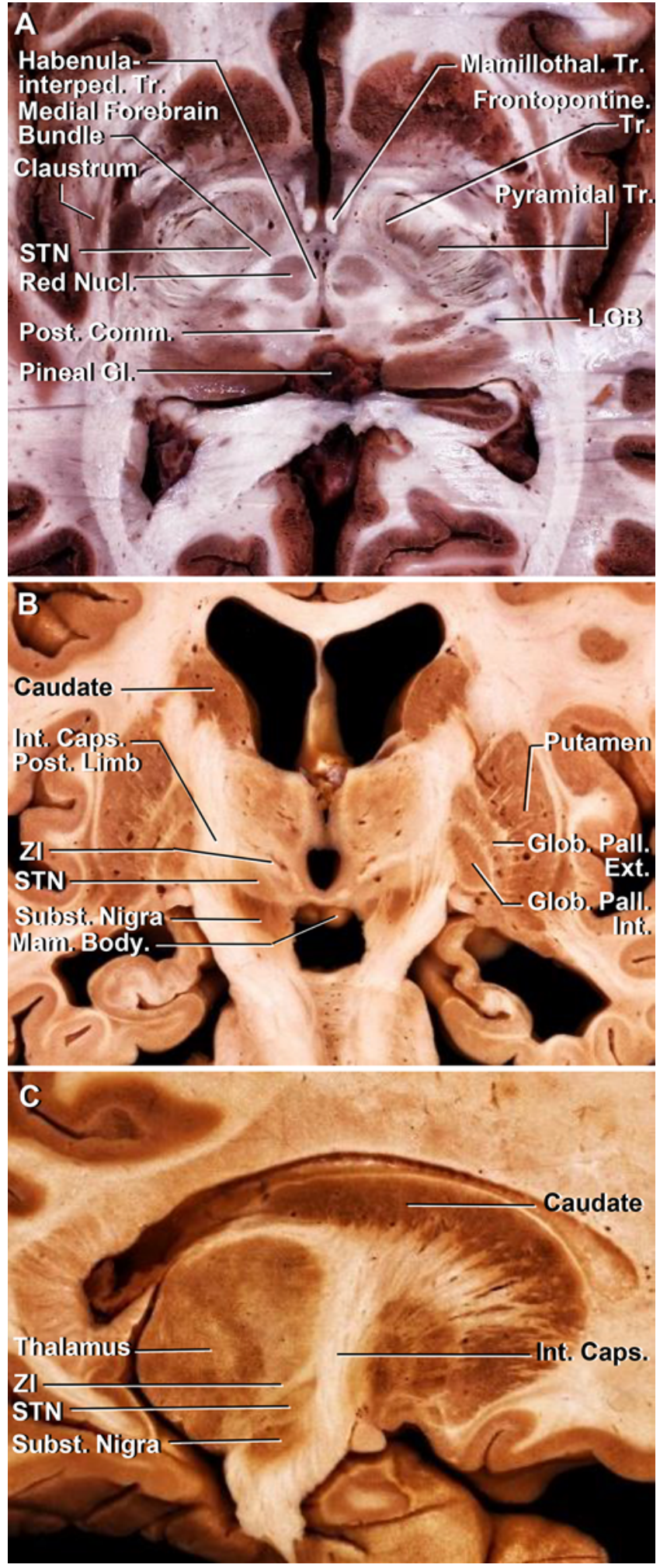

FIG. 7. Axial, coronal, and sagittal anatomical sections of the diencephaIon at different levels to display the STN and neighboring structures in the human brain. A: A midbrain axial cut through the upper edge of the superior colliculi has been performed to show the STN and its relationships. The STN is located anterolateral to the red nucleus and posteromedial to the corticospinal tract. The MFB is medial to the STN at this level. B: A coronal cut has been performed at the level of the central rolandic sulcus of the lateral cerebral hemisphere. FIG. 7. (continued) $\rightarrow$
FIG. 7. The coronal cut corresponds to the anterior edge of the mammillary bodies medially. The STN is located between the ZI and substantia nigra with its almond-like shape just medial to the IC. The red nucleus is situated more posteriorly and cannot be seen at this section. C: A sagittal cut has been performed to show STN and its relationships. The $\mathrm{Zl}$ and substantia nigra spread to the posterior subthalamic region, from superior and from inferior, respectively. Interped. = interpeduncular. Copyright Necmettin Tanriover. Published with permission. Figure is available in color online only.

region, run anteriorly lateral to the red nucleus and medial to the STN (Fig. 9C-G). The medial forebrain bundle (MFB) is exposed by removing the STN medially and moving in an inferior direction (Figs. $2 \mathrm{~F}$ and $9 \mathrm{C}-\mathrm{G}$ ). The MFB, a longitudinal midbrain limbic pathway formed by densely packed neurons projecting from the septal region to the ventral tegmental area, invades several forebrain structures, including the lateral and medial hypothalamus, sublenticular region, lateral and medial preoptic regions, diagonal band, septal nuclei, ventral pallidum, and the bed nuclei of the stria terminalis, in addition to other limbic system-related structures. The MFB lies lateral to the red nucleus on axial planes and at the level of the red nucleus on sagittal planes. The MFB is located lateral to the mammillothalamic tract, medial to the substantia nigra, and slightly inferomedial to the STN (Figs. 2F and 9C-G).

Among the laterally located structures, the optic tract runs inferolateral to the STN along its path toward the LGB. The optic tract lies inferior to the GPi, IC, and ansa lenticularis. The corticospinal fibers run along the cerebral peduncle ventral and then lateral to the STN, before ascending and merging into other components of the IC (Fig. 2E). The medial lemniscus ascends from the gracile and cuneate tubercles and runs superiorly dorsal to the cerebral peduncle. Before terminating in the thalamus, the medial lemniscus ascends dorsal to the substantia nigra, ventrolateral to the red nucleus, and posteroinferior to the STN. At the level of the STN, the medial lemniscus is located posterior to the STN. The bundle of prelemniscal radiations courses in front of the medial lemniscus lateral to the red nucleus and medial to the STN (Fig. 9C-G).

This study also examined the sectional anatomy of the STN and related structures in brain specimens through axial, coronal, and sagittal planes (Fig. 7A-C). The STN, with its almond shape, lies at an oblique angle to the anteroposterior axis of the brainstem just above the substantia nigra (Fig. 7A-C). Although in dissections the STN and substantia nigra were clearly demarcated by color, we were not able to discriminate between these two structures in MRI due to similar signal intensities on the sequences. As a result, we built a 3D terrain model of the subthalamic area encircling the STN, which includes the putamen, GPi, globus pallidus externus (GPe), IC, caudate nucleus laterally, substantia nigra inferiorly, and ZI and red nucleus medially. We also described the relationship of the medial lemniscus, oculomotor nerve fibers, and the MFB with the STN by using MR tractography with the 3D STN model (Figs. 6A and B, 7A-D, 8A-F, 9A-F, and 10A-D). To verify our dissection results, we correlated anatomical dissection data with those obtained by the MRI of the same specimen with the aid of a neuronavigation system (Fig. 11A-F). 

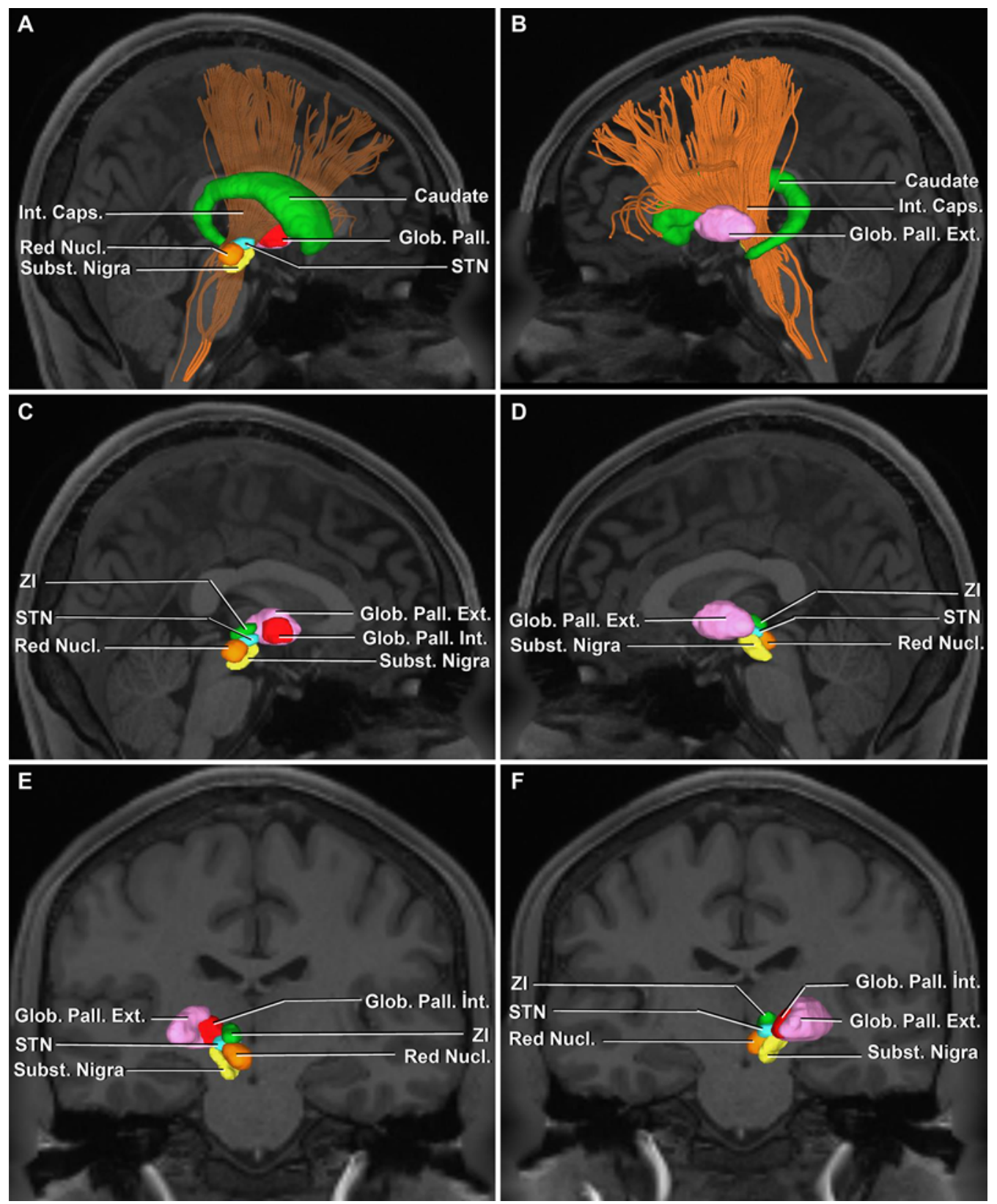

FIG. 8. 3D MRI reconstruction of neighboring anatomical structures associated with the STN and their correlations with the related white matter tracts through diffusion tensor imaging. A: Medial view; the STN (turquoise blue) is located superior to the substantia nigra (yellow), lateral to the red nucleus (orange), and medial to the IC and GPi. The IC fibers ascend from the inferior aspect of the STN and over its lateral surface and pass lateral to the caudate nucleus (green) at the level of the insula. B: Lateral view; the IC fibers ascend from the inferior aspect of the STN and over its lateral surface, medial to the globus pallidus (pink), and pass lateral to the caudate nucleus at the level of the insula. C: Medial 3D MRI view of the STN (turquoise blue), red nucleus (orange), ZI (green), substantia nigra (yellow), GPi (red), and GPe (pink). The red nucleus is located inferomedial to the STN with its spheroid shape. The ZI covers the superior surface of the STN and reaches its posterior border. The substantia nigra is located inferior to the red nucleus and the STN and also spreads toward the posterior territory of the STN. D: Lateral view of 3D MRI reconstruction of the STN (turquoise blue), red nucleus (orange), ZI (green), substantia nigra (yellow), and GPe (pink). The Zl extends from midline to the inferolateral border of the STN lying under the subthalamic and red nuclei. E: Anterior view of 3D MRI reconstruction of the STN (turquoise blue), red nucleus (orange), ZI (green), substantia nigra (yellow), GPi (red), and GPe (pink). The STN is surrounded by red nucleus posteromedially, by ZI (green) superiorly, and by substantia nigra inferoposteriorly. F: Posterior view of 3D MRI reconstruction of the STN (turquoise blue), red nucleus (orange), ZI (yellow), substantia nigra, GPi (red), and GPe (pink). The ZI from superior and substantia nigra from inferior extend to the posterior subthalamic area. Figure is available in color online only. 

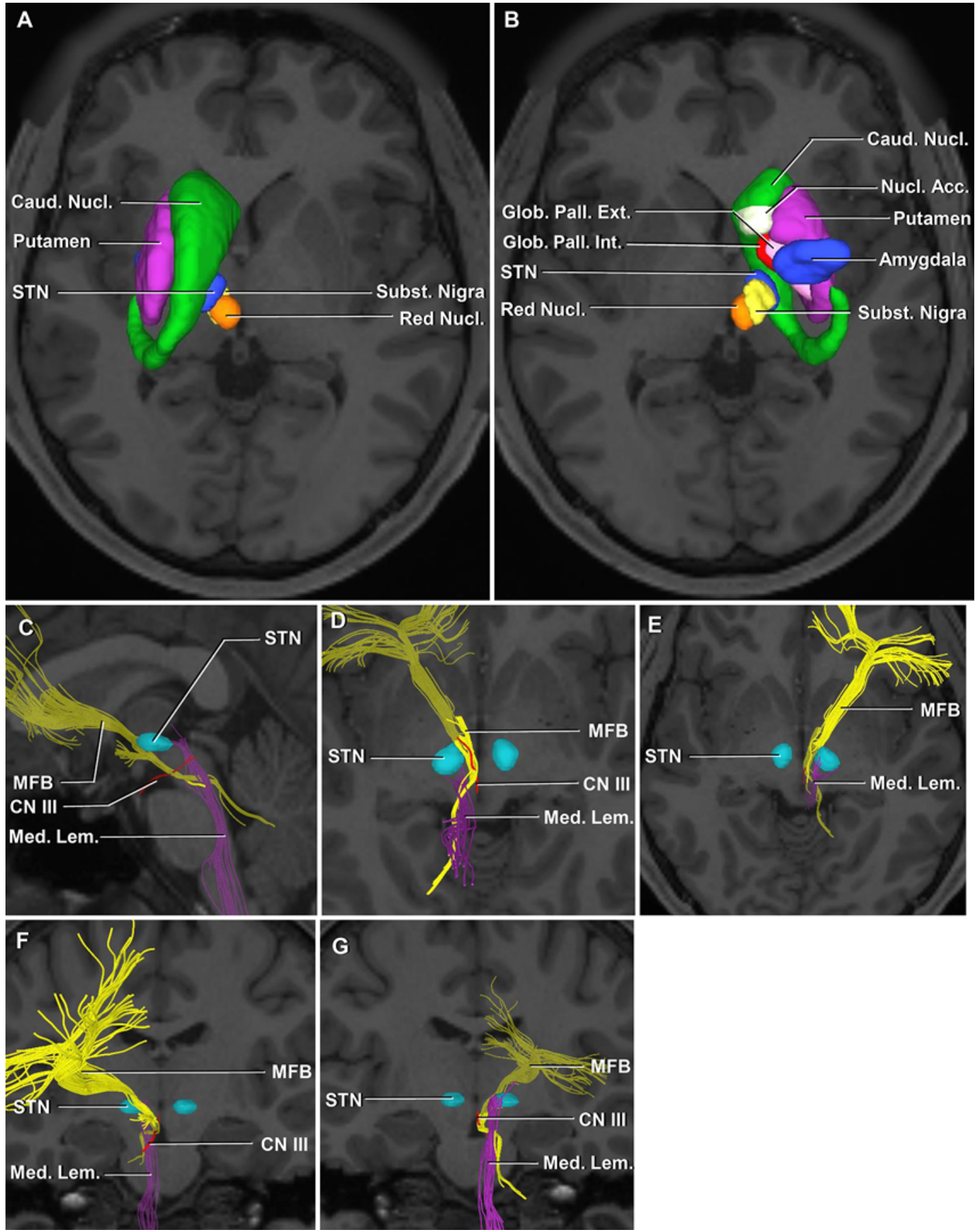

FIG. 9. 3D MRI reconstruction of neighboring anatomical structures associated with the STN and their correlations with the related white matter tracts through diffusion tensor imaging. A: Superior view of 3D MRI reconstruction of the STN (dark blue), red nucleus (orange), substantia nigra (yellow), caudate nucleus (green), and putamen (fuchsia). B: Inferior view of 3D MRI reconstruction of the STN (dark blue), red nucleus (orange), substantia nigra (yellow), GPi (red), GPe (pink), amygdala (dark blue), caudate nucleus (green), and putamen (fuchsia). C: Lateral view of 3D MRI reconstruction of the STN (turquoise blue) and tractography of the MFB (yellow), medial lemniscus (purple), and oculomotor nerve fibers (red). The medial lemniscus is located posterior to the STN. The MFB is located inferomedial to the STN and oculomotor nerve fibers are located inferomedial to the STN. D: Superior view of panel D. The medial lemniscus ascends posteroinferior to the STN. The oculomotor nerve fibers are medial to the STN. The medial forebrain bundle lies slightly inferomedial to the STN. E: Inferior view of panel D. F: Anterior view of panel D. G: Posterior view of panel $\mathrm{D}$. Figure is available in color online only. 

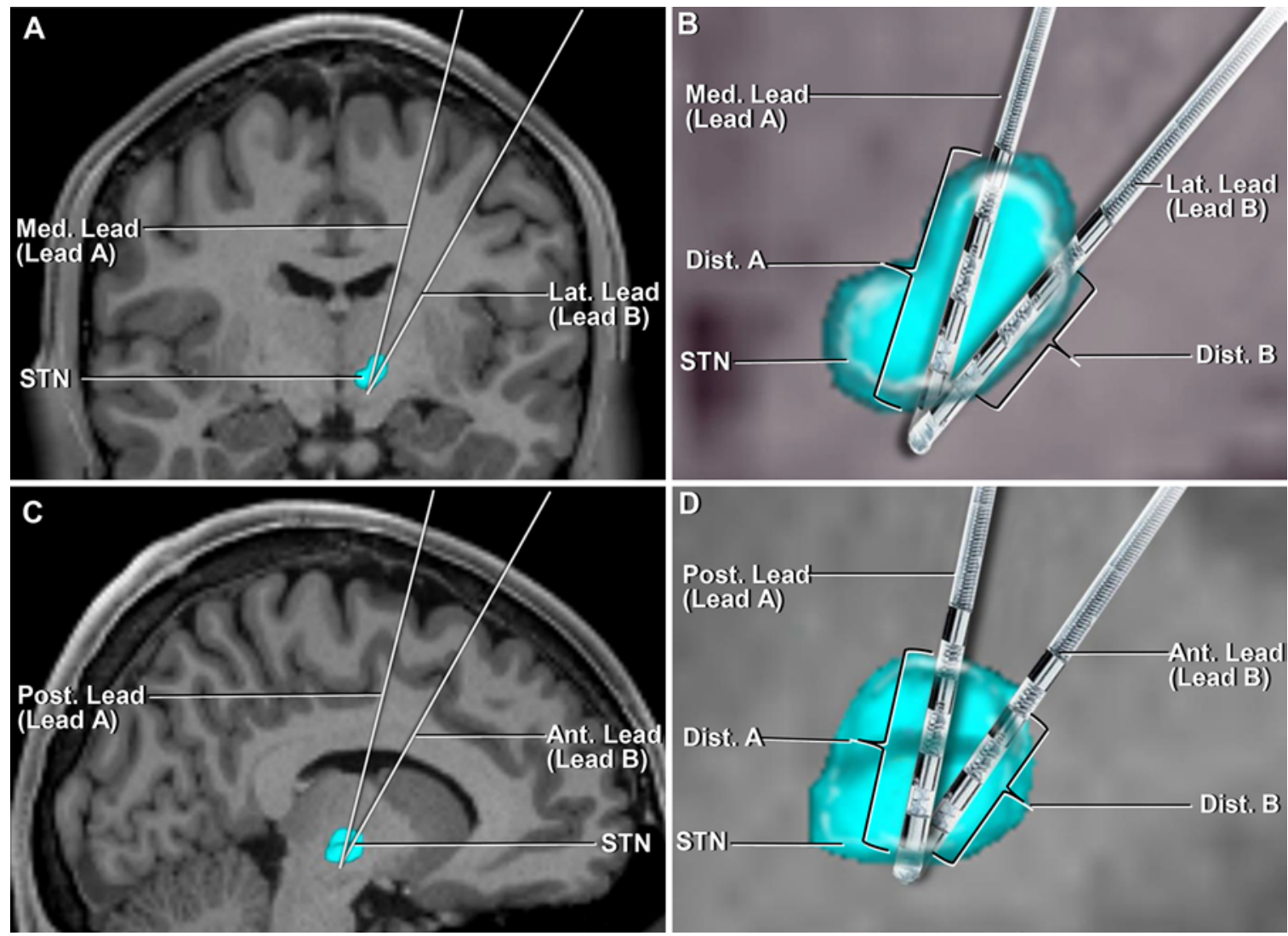

FIG. 10. Demonstration of lead insertion to the STN from different angles. A: Coronal T1-weighted image obtained at the STN level with 3D STN modeling and depiction of a DBS lead insertion from 2 different angles in the coronal plane. Demonstration that changing the entry angle without changing the target can significantly alter the position of the implanted DBS contacts. Lead B would likely have excessively low thresholds for capsular side effects. B: Enlarged view of panel A. C: Sagittal T1-weighted image obtained at the STN level with 3D STN modeling and depiction of a DBS lead insertion from 2 different angles in the sagittal plane. Demonstration that changing the entry angle without changing the target can significantly alter the position of the implanted DBS contacts. Lead B would likely have excessively low thresholds for capsular side effects. D: Enlarged view of panel C. Dist. = distance. Figure is available in color online only.

\section{Discussion}

The STN plays a fundamental role within the basal ganglia-thalamocortical motor, associative, and limbic circuits. The nucleus has 3 subdivisions and has been functionally divided into the sensorimotor (dorsolateral), limbic (medial), and cognitive-associative (ventromedial) segments. ${ }^{21,22,33}$ The dorsolateral sensorimotor region of the STN in Parkinson's disease is the generally accepted target for DBS treatment of motor symptoms, such as tremor and bradykinesia. ${ }^{11,13,15,28,33,42,44,56}$ STN stimulation has also been explored as an option to treat refractory epilepsy and obsessive-compulsive disorder. ${ }^{11,13,15,28,56}$ Precise and thorough identification of the 3D anatomy of the STN and related structures is a prerequisite for all of these DBS procedures. Safe and effective DBS procedures targeting the STN also depend on a thorough understanding of the 3D anatomy of the region, which is critical for proper assessment of both outcomes and adverse effects. ${ }^{7}$ However, even with the best available imaging techniques for displaying brain anatomy in vivo, the peri-STN area is still poorly visualized. . $^{25,30,46,49}$

Several approaches have been described to optimize STN targeting. DBS centers predominantly use the con- cept of a "2-stage method" for accurate and clinically effective positioning of electrodes. The first stage consists of anatomical target location, and the second stage employs neurophysiological feedback to confirm the appropriate target selection. Anatomical targeting is generally accomplished using one of 3 alternative techniques: indirect, direct, or hybrid targeting. Indirect targeting relies upon coordinates obtained from standard brain atlases, even though anatomical and histological studies demonstrate unequivocally that the sizes and shapes of the STN and adjacent structures vary among individuals. ${ }^{2,3,50,65-67}$ This variability supports the use of direct targeting, which requires STN visualization on targeting MRI. Finally, a combined method-hybrid targeting-has been described, which augments the accuracy of indirect targeting using direct STN visualization. Neurophysiological confirmation is generally accomplished with microelectrode recording and macrostimulation techniques., ${ }^{9,18}$ If the ideal target can be reached with effective anatomical targeting, then the time required for neurophysiological confirmation will be strikingly shortened..$^{57}$

Regardless of the method used, understanding the 3D anatomical relations of the STN region is mandatory for 

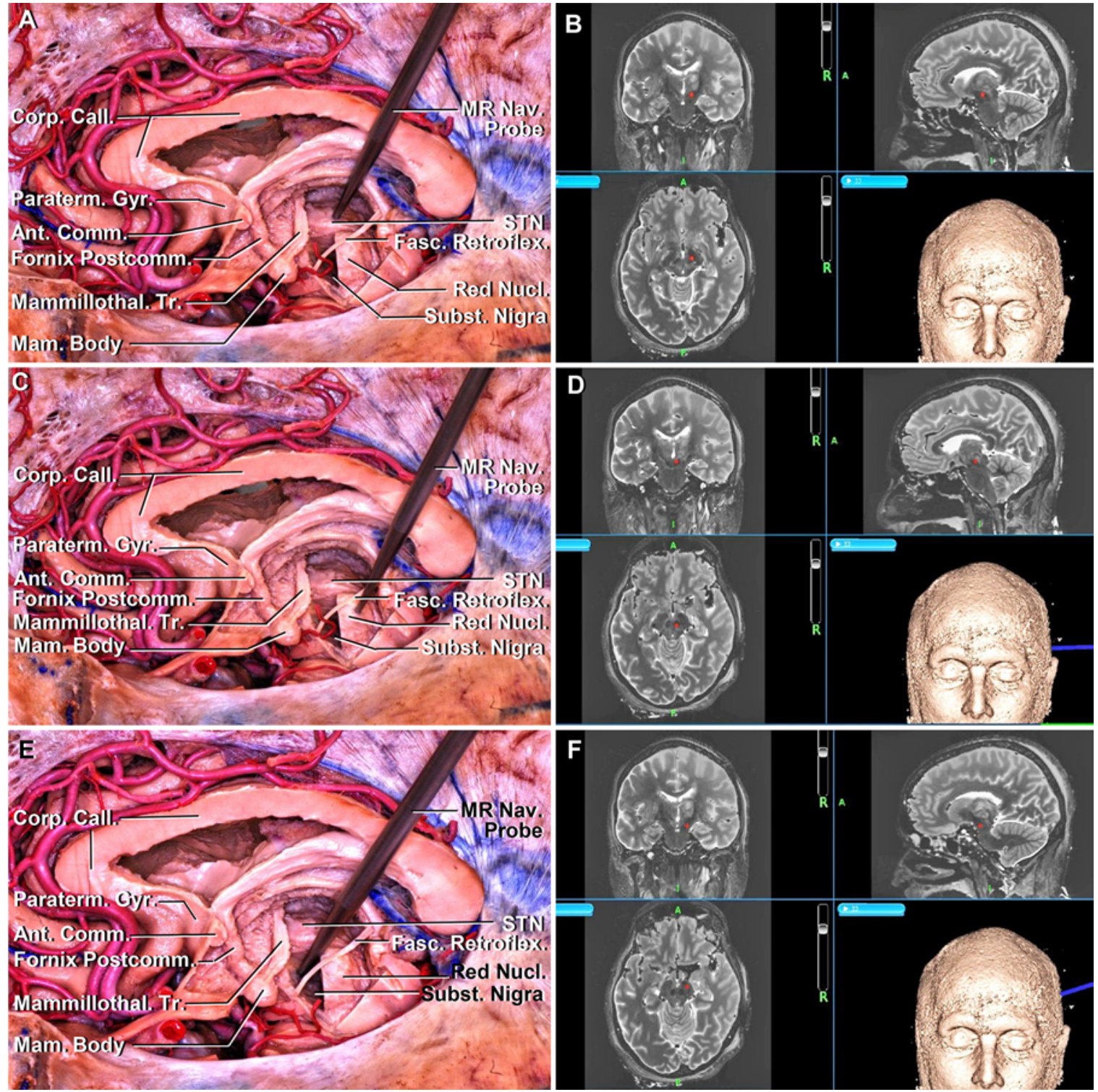

FIG. 11. Medial to lateral fiber dissection of the diencephalon on a silicone-injected cadaveric head with the aid of MR navigation. A: The ventral part of the thalamus and ventral tegmental area have been removed to expose the STN and red nucleus. The anterior part of the red nucleus has been removed to expose the substantia nigra. The navigation probe shows the superomedial part of the STN. The STN is located lateral to the red nucleus and superior to the substantia nigra. B: MRI-based stereotactic localization with the probe on the medial aspect of the STN on coronal, sagittal, and axial T2 MR sections of the same specimen. C: The navigation probe on the superomedial aspect of the red nucleus. The red nucleus is located posterior to the mammillothalamic tract and mammillary body, medial to the STN, and superior to the substantia nigra. D: Stereotactic localization of the superomedial aspect of the red nucleus. E: The navigation probe on the medial aspect of the substantia nigra. The substantia nigra lies ventral to the red nucleus and the STN. F: Stereotactic localization of the medial aspect of the substantia nigra. Nav. = navigation; Paraterm. $=$ paraterminal. Panels A, C, and E: Copyright Necmettin Tanriover. Published with permission. Figure is available in color online only.

optimal surgical results - and for minimizing unwelcome side effects. To the best of our knowledge, no prior study has attempted to clarify the surgical anatomy of the subthalamic region using precise anatomical fiber dissection, with special emphasis on the STN and related structures. We used Klingler's fiber dissection method, currently the gold standard to validate tractography results in the human brain, with concordance to diffusion tractography using a GQI model. The methods, however, have some limitations, including inevitable shrinkage and distortion effects of the preparation process on the postmortem brains, the limited exposure of the origin and termination areas of the fiber tracts in regard to the cortex, and the inability to detect crossing fibers that cannot be studied completely in the same dissection. ${ }^{68}$ Nevertheless, the current study is the first to correlate the results of STN fiber dissection with those of 3D MRI reconstruction and tractography using neuronavigation. As a result, we were able to build a 3D 
terrain model of the subthalamic area encircling STN and clarify its anatomical relationships with the putamen, GPi, GPe, IC, caudate nucleus, substantia nigra, ZI, and red nucleus.

Stimulation-induced adverse effects, although typically transient and alleviated by adjusting the parameters of the stimulator, remain a challenge during STN DBS and can be understood in relation to the electrode's anatomical location. Suboptimal electrode location is not the only explanation for these adverse effects, since the STN is a small structure and the delivery of excessive current to even a well-placed electrode can result in the spreading of current into adjacent functional circuitry. Furthermore, previous studies have divided stimulation-related adverse effects into 2 categories: capsular (signs or symptoms considered to be caused by current spread to the IC) and noncapsular. Our study sheds light on the adverse effects commonly seen in association with STN stimulation and their anatomical substrates. ${ }^{35,52,53}$

\section{Capsular Effects}

Studies of the effects of stimulation on neural tissue suggest that axons are more readily activated than neuronal cell bodies. Accordingly, capsular effects are commonly observed with high-frequency stimulation. Muscle contractions in the limbs, torso, and head can be detected when the volume of brain tissue activation extends to the corticospinal tract within the IC, which runs from the ventral to the anterolateral aspect of the STN. ${ }^{16}$ Facial pulling, dysarthria, and eye deviation may all be related to the activation of the corticobulbar and corticomesencephalic fibers within the IC. With regard to facial muscle contraction during STN DBS, Gorgulho et al. described the most common electrode localization as the anterolateral part of the superior border of the nucleus. ${ }^{17}$ Tsuboi et al. reported that dysarthria occurs mainly due to alterations of the laryngeal muscles when the electrode localization is lateral or superior to the STN. ${ }^{55}$ Disturbance of frontal eye field projections, running within the posterior limb of the IC and the medial portion of the cerebral peduncle, at the lateral anterosuperior border of the STN is presumably responsible for conjugate eye deviation during stimulation. ${ }^{47}$ According to our dissections, the IC has a close relationship with the lateral anterior and inferior borders of the STN as it descends toward the pons. In the event that capsular signs (e.g., involuntary muscle contractions, dysarthria, conjugate eye deviation) occur during stimulation testing, repositioning of the electrode medially or inferomedially may remediate these adverse effects.

\section{Noncapsular Effects}

Noncapsular effects, such as unilateral eye deviation, heat sensation, sweating, and dizziness, are typically observed when stimulation extends medially and posteriorly to the STN. ${ }^{31,35,51,59}$ These regions correspond to the hypothalamus, medial lemniscus, and red nucleus according to our dissections.

Activation of the medial lemniscus in the posterior subthalamic area, which also includes the ZI and part of the substantia nigra immediately posterior to the STN, can produce contralateral paresthesia (with symptoms such as tingling or electrical sensations). ${ }^{51}$

Posterior subthalamic area DBS has been advocated as an effective treatment for intention tremor and for tremordominant Parkinson's disease. Observing paresthesia with high-frequency macrostimulation of the posterior subthalamic area may be helpful for target selection in these cases. ${ }^{8,10,24}$ Eye adduction and diplopia can be observed with stimulation of the oculomotor nerve (cranial nerve [CN] III) fibers through their intramesencephalic course, as they pass lateral to the red nucleus and medial to the STN after leaving the CN III nucleus. ${ }^{48,51}$ Experiencing an eye deviation during DBS of the STN may suggest redundant medial and posterior trajectory of the electrodes and close proximity to the CN III fibers and may require a more lateral trajectory. Autonomic side effects are subjective and nonspecific symptoms, including sweating, malaise, dizziness, and heat sensation, that have been attributed to activation of the ZI, red nucleus, or connections between the tegmentum and hypothalamus. ${ }^{51}$ Our dissections reveal that the rostral part of the ZI extends over the dorsal and medial surface of the STN, and its caudal portion lies posteromedial to the STN. The red nucleus, on the other hand, lies posterior and inferomedial to the STN. The dorsal longitudinal fasciculus and MFB, which constitute the medial and lateral hypothalamic areas, respectively, seem most likely to be the anatomical connections responsible for secondary autonomic side effects of STN DBS. The association of nonspecific autonomic symptoms with stimulation of structures situated medially and inferiorly to the STN suggests that repositioning electrode contacts more laterally and superiorly might reduce these symptoms.

A growing body of literature suggests that a number of subtle neuropsychological and neurobehavioral adverse events are associated with STN stimulation. In fact, this concept is still controversial and these events may be in part due to changes in dopaminergic medication or progression of the disease..$^{14,54,59}$ Affective dysregulation related to STN stimulation can manifest in a wide range of symptoms and signs, including hypomania and mania, general anxiety, impulse control disorder, major depression, acute psychosis with delusions, changes in personality, hypersexuality, or drug dependence. ${ }^{14,25,27,52,54}$ This collection of complex neuropsychological and neurobehavioral adverse effects is presumed to be associated with medial or ventral stimulation corresponding to the limbic region of the STN. In addition, the medially located MFB on the lateral part of the hypothalamus and the ventrally located substantia nigra may play a role in these psychological and behavioral adverse effects. ${ }^{20,51,58,59,62,63}$

Stimulation-related cognitive adverse effects are variable and include deterioration in verbal fluency, memory, executive functioning, mental speed, response inhibition, and attention. ${ }^{19,31,40,42,45,52,60}$ Similar to psychological and behavioral effects, cognitive adverse effects are associated with ventrally and medially located electrodes that spread current into the cognitive-associative region of the STN. 19,40,42 These ventromedial regions contain the nonmotor circuits within the STN. ${ }^{19,38,40,42}$ Based on these data, we suggest that targeting the sensorimotor (dorsolateral) region of the STN is necessary to avoid cognitive ad- 
verse events associated with limbic and associative STN circuits. Furthermore, it has been suggested that penetration of the caudate nucleus by the microelectrodes during DBS surgery might be associated with a decline in neuropsychological functioning. ${ }^{62}$ Morishita et al. and Isler et al., however, found no significant difference in cognitive decline between caudate-penetrated and caudate-spared groups. 5,23,36 According to our dissections, preserving the integrity of the caudate nucleus may be possible with a more anterolateral trajectory. We also speculate that cognitive decline may be related, at least in part, to disruption or inadvertent activation of structures en route to the STN, including subcortical white matter tracts (SLF), the anterior portion of the corona radiata, the anterior thalamic peduncle, and callosal fibers, in addition to the thalamus.

Our anatomical study also suggests that by exploiting 3D anatomical knowledge of the STN, the surgeon can adjust the trajectory of a DBS lead to increase the length of an electrode traversing the STN, resulting in the positioning of more electrode contacts within the nucleus.

We believe that effective targeting of the STN requires precise anatomical knowledge of the area, and we hope that this report will clarify this complex anatomy for DBS practitioners. Our ability to define the STN and related structures on MR images may be enhanced by this study, which would enable more effective direct targeting of the STN.

\section{Conclusions}

Familiarity with the complex 3D anatomy of the STN and peri-subthalamic area should result in more effective targeting of the STN for DBS therapy. Therapeutic benefit can be maximized and adverse effects diminished through the application of a detailed understanding of the functional neuroanatomy of the target region. We believe that the combination of meticulous anatomical dissections of the STN region with detailed discussions of pertinent anatomo-functional relationships included in this study will provide DBS practitioners with a more sophisticated understanding of this important brain region and empower them to improve the outcomes of STN DBS for their patients.

\section{Acknowledgments}

We are grateful to Albert L. Rhoton Jr., MD, for his invaluable guidance and advice over the years and for his support with this manuscript, and we also deeply appreciate the continuous support provided by Robin Barry in preparation of the figures and by Jessica Striley with the manuscript.

\section{References}

1. Alarcon C, de Notaris M, Palma K, Soria G, Weiss A, Kassam A, et al: Anatomic study of the central core of the cerebrum correlating 7-T magnetic resonance imaging and fiber dissection with the aid of a neuronavigation system. Neurosurgery 10 (Suppl 2):294-304, 2014

2. Bardinet E, Bhattacharjee M, Dormont D, Pidoux B, Malandain G, Schüpbach M, et al: A three-dimensional histological atlas of the human basal ganglia. II. Atlas deformation strategy and evaluation in deep brain stimulation for Parkinson disease. J Neurosurg 110:208-219, 2009
3. Bardinet E, Dormont D, Malandain G, Bhattacharjee M, Pidoux B, Saleh C, et al: Retrospective cross-evaluation of an histological and deformable 3D atlas of the basal ganglia on series of Parkinsonian patients treated by deep brain stimulation. Med Image Comput Comput Assist Interv 8:385-393, 2005

4. Baydin S, Gungor A, Tanriover N, Baran O, Middlebrooks $\mathrm{EH}$, Rhoton AL Jr: Fiber tracts of the medial and inferior surfaces of the cerebrum. World Neurosurg 98:34-49, 2017

5. Baydin S, Gungor A, Tanriover N, Rhoton LA Jr: In reply: Microsurgical and fiber tract anatomy of the nucleus accumbens. Operative Neurosurg 12:E396-E397, 2016 (Letter)

6. Benabid AL, Benazzouz A, Hoffmann D, Limousin P, Krack P, Pollak P: Long-term electrical inhibition of deep brain targets in movement disorders. Mov Disord 13 (Suppl 3):119-125, 1998

7. Benabid AL, Chabardes S, Mitrofanis J, Pollak P: Deep brain stimulation of the subthalamic nucleus for the treatment of Parkinson's disease. Lancet Neurol 8:67-81, 2009

8. Blomstedt P, Fytagoridis A, Åström M, Linder J, Forsgren L, Hariz MI: Unilateral caudal zona incerta deep brain stimulation for Parkinsonian tremor. Parkinsonism Relat Disord 18:1062-1066, 2012

9. Bronstein JM, Tagliati M, Alterman RL, Lozano AM, Volkmann J, Stefani A, et al: Deep brain stimulation for Parkinson disease: an expert consensus and review of key issues. Arch Neurol 68:165, 2011

10. Carrillo-Ruiz JD, Velasco F, Jimènez F, Castro G, Velasco AL, Hernández JA, et al: Bilateral electrical stimulation of prelemniscal radiations in the treatment of advanced Parkinson's disease. Neurosurgery 62:347-359, 2008

11. Chabardès S, Polosan M, Krack P, Bastin J, Krainik A, David $\mathrm{O}$, et al: Deep brain stimulation for obsessive-compulsive disorder: subthalamic nucleus target. World Neurosurg 80:31. e1-31.e8, 2013

12. Choi CY, Han SR, Yee GT, Lee CH: Central core of the cerebrum. J Neurosurg 114:463-469, 2011

13. Cleary DR, Ozpinar A, Raslan AM, Ko AL: Deep brain stimulation for psychiatric disorders: where we are now. Neurosurg Focus 38(6):E2, 2015

14. Coenen VA, Honey CR, Hurwitz T, Rahman AA, McMaster J, Bürgel U, et al: Medial forebrain bundle stimulation as a pathophysiological mechanism for hypomania in subthalamic nucleus deep brain stimulation for Parkinson's disease. Neurosurgery 64:1106-1115, 2009

15. de Koning PP, Figee M, van den Munckhof P, Schuurman PR, Denys D: Current status of deep brain stimulation for obsessive-compulsive disorder: a clinical review of different targets. Curr Psychiatry Rep 13:274-282, 2011

16. Ferrarin M, Carpinella I, Rabuffetti M, Rizzone M, Lopiano L, Crenna P: Unilateral and bilateral subthalamic nucleus stimulation in Parkinson's disease: effects on EMG signals of lower limb muscles during walking. IEEE Trans Neural Syst Rehabil Eng 15:182-189, 2007

17. Gorgulho AA, Shields DC, Malkasian D, Behnke E, Desalles AA: Stereotactic coordinates associated with facial musculature contraction during high-frequency stimulation of the subthalamic nucleus. J Neurosurg 110:1317-1321, 2009

18. Güngör A, Baydin S, Middlebrooks EH, Tanriover N, Isler C, Rhoton AL Jr: The white matter tracts of the cerebrum in ventricular surgery and hydrocephalus. J Neurosurg 126:945-971, 2017

19. Haegelen C, García-Lorenzo D, Le Jeune F, Péron J, Gibaud B, Riffaud L, et al: SPECT and PET analysis of subthalamic stimulation in Parkinson's disease: analysis using a manual segmentation. J Neurol 257:375-382, 2010

20. Hana A, Hana A, Dooms G, Boecher-Schwarz H, Hertel F: Visualization of the medial forebrain bundle using diffusion tensor imaging. Front Neuroanat 9:139, 2015 
21. Haynes WI, Haber SN: The organization of prefrontal-subthalamic inputs in primates provides an anatomical substrate for both functional specificity and integration: implications for basal ganglia models and deep brain stimulation. J Neurosci 33:4804-4814, 2013

22. Hendelman W: Atlas of Functional Neuroanatomy. Boca Raton: CRC Press, 2005

23. Isler C, Albi A, Schaper FL, Temel Y, Duits A: Neuropsychological outcome in subthalamic nucleus stimulation surgeries with electrodes passing through the caudate nucleus. Stereotact Funct Neurosurg 94:413-420, 2016

24. Kitagawa M, Murata J, Uesugi H, Kikuchi S, Saito H, Tashiro $\mathrm{K}$, et al: Two-year follow-up of chronic stimulation of the posterior subthalamic white matter for tremor-dominant Parkinson's disease. Neurosurgery 56:281-289, 2005

25. Kitajima M, Hirai T, Shigematsu Y, Uetani H, Iwashita K, Morita K, et al: Comparison of 3D FLAIR, 2D FLAIR, and 2D T2-weighted MR imaging of brain stem anatomy. AJNR Am J Neuroradiol 33:922-927, 2012

26. Kleiner-Fisman G, Fisman DN, Sime E, Saint-Cyr JA, Lozano AM, Lang AE: Long-term follow up of bilateral deep brain stimulation of the subthalamic nucleus in patients with advanced Parkinson disease. J Neurosurg 99:489-495, 2003

27. Kleiner-Fisman G, Herzog J, Fisman DN, Tamma F, Lyons KE, Pahwa R, et al: Subthalamic nucleus deep brain stimulation: summary and meta-analysis of outcomes. Mov Disord 21 (Suppl 14):S290-S304, 2006

28. Klinger NV, Mittal S: Clinical efficacy of deep brain stimulation for the treatment of medically refractory epilepsy. Clin Neurol Neurosurg 140:11-25, 2016

29. Klingler J: Erleichterung der makroskopischen Praeparation des Gehirns durch den Gefrierprozess. Schweiz Arch Neurol Psychiatr 36:247-256, 1935

30. Kocabicak E, Alptekin O, Ackermans L, Kubben P, Kuijf M, Kurt E, Esselink R, Temel Y: Is there still need for microelectrode recording now the subthalamic nucleus can be well visualized with high field and ultrahigh MR imaging? Front Integr Neurosci 9:46, 2015

31. Krack P, Fraix V, Mendes A, Benabid AL, Pollak P: Postoperative management of subthalamic nucleus stimulation for Parkinson's disease. Mov Disord 17 (Suppl 3):S188-S197, 2002

32. Lozano AM: Deep brain stimulation for Parkinson's disease. Parkinsonism Relat Disord 7:199-203, 2001

33. Manes JL, Parkinson AL, Larson CR, Greenlee JD, Eickhoff SB, Corcos DM, et al: Connectivity of the subthalamic nucleus and globus pallidus pars interna to regions within the speech network: a meta-analytic connectivity study. Hum Brain Mapp 35:3499-3516, 2014

34. Massey LA, Miranda MA, Zrinzo L, Al-Helli O, Parkes HG, Thornton JS, et al: High resolution MR anatomy of the subthalamic nucleus: imaging at $9.4 \mathrm{~T}$ with histological validation. Neuroimage 59:2035-2044, 2012

35. Matias CM, Mehanna R, Cooper SE, Amit A, Lempka SF, Silva D, et al: Correlation among anatomic landmarks, location of subthalamic deep brain stimulation electrodes, stimulation parameters, and side effects during programming monopolar review. Neurosurgery 11 (Suppl 2):99-109, 2015

36. Morishita T, Okun MS, Jones JD, Foote KD, Bowers D: Cognitive declines after deep brain stimulation are likely to be attributable to more than caudate penetration and lead location. Brain 137:e274, 2014

37. Nakano N, Taneda M, Watanabe A, Kato A: Computed three-dimensional atlas of subthalamic nucleus and its adjacent structures for deep brain stimulation in Parkinson's disease. ISRN Neurol 2012:592678, 2012

38. Nieuwenhuys R, Voogd J, van Huijzen C: The Human Central Nervous System. A Synopsis and Atlas. New York: Springer, 2005, p 247
39. Nowinski WL: Towards construction of an ideal stereotactic brain atlas. Acta Neurochir (Wien) 150:1-14, 2008

40. Okun MS, Fernandez HH, Wu SS, Kirsch-Darrow L, Bowers D, Bova F, et al: Cognition and mood in Parkinson's disease in subthalamic nucleus versus globus pallidus interna deep brain stimulation: the COMPARE trial. Ann Neurol 65:586595, 2009

41. Okun MS, Foote KD: Parkinson's disease DBS: what, when, who and why? The time has come to tailor DBS targets. Expert Rev Neurother 10:1847-1857, 2010

42. Parsons TD, Rogers SA, Braaten AJ, Woods SP, Tröster AI: Cognitive sequelae of subthalamic nucleus deep brain stimulation in Parkinson's disease: a meta-analysis. Lancet Neurol 5:578-588, 2006

43. Richter EO, Hoque T, Halliday W, Lozano AM, Saint-Cyr JA: Determining the position and size of the subthalamic nucleus based on magnetic resonance imaging results in patients with advanced Parkinson disease. J Neurosurg 100:541-546, 2004

44. Rocchi L, Carlson-Kuhta P, Chiari L, Burchiel KJ, Hogarth P, Horak FB: Effects of deep brain stimulation in the subthalamic nucleus or globus pallidus internus on step initiation in Parkinson disease: laboratory investigation. J Neurosurg 117:1141-1149, 2012

45. Schroeder U, Kuehler A, Lange KW, Haslinger B, Tronnier VM, Krause M, et al: Subthalamic nucleus stimulation affects a frontotemporal network: a PET study. Ann Neurol 54:445-450, 2003

46. Senova S, Hosomi K, Gurruchaga JM, Gouello G, Ouerchefani N, Beaugendre Y, et al: Three-dimensional SPACE fluidattenuated inversion recovery at $3 \mathrm{~T}$ to improve subthalamic nucleus lead placement for deep brain stimulation in Parkinson's disease: from preclinical to clinical studies. J Neurosurg 125:472-480, 2016

47. Shields DC, Gorgulho A, Behnke E, Malkasian D, DeSalles AA: Contralateral conjugate eye deviation during deep brain stimulation of the subthalamic nucleus. J Neurosurg 107:37-42, 2007

48. Spiegel EA, Wycis HT, Szekely EG, Soloff L, Adams J, Gildenberg P, et al: Stimulation of Forel's field during stereotaxic operations in the human brain. Electroencephalogr Clin Neurophysiol 16:537-548, 1964

49. Sudhyadhom A, Haq IU, Foote KD, Okun MS, Bova FJ: A high resolution and high contrast MRI for differentiation of subcortical structures for DBS targeting: the Fast Gray Matter Acquisition T1 Inversion Recovery (FGATIR). Neuroimage 47 (Suppl 2):T44-T52, 2009

50. Talairach J, Tournoux P: Referentially Oriented Cerebral MRI Anatomy: An Atlas of Stereotaxic Anatomical Correlations for Gray and White Matter. New York: Thieme, 1993

51. Tamma F, Caputo E, Chiesa V, Egidi M, Locatelli M, Rampini P, et al: Anatomo-clinical correlation of intraoperative stimulation-induced side-effects during HF-DBS of the subthalamic nucleus. Neurol Sci 23 (Suppl 2):S109-S110, 2002

52. Temel Y: Subthalamic nucleus stimulation in Parkinson's disease: the other side of the medallion. Exp Neurol 211:321323,2008

53. Tommasi G, Krack P, Fraix V, Le Bas JF, Chabardes S, Benabid AL, et al: Pyramidal tract side effects induced by deep brain stimulation of the subthalamic nucleus. J Neurol Neurosurg Psychiatry 79:813-819, 2008

54. Tsai ST, Lin SH, Lin SZ, Chen JY, Lee CW, Chen SY: Neuropsychological effects after chronic subthalamic stimulation and the topography of the nucleus in Parkinson's disease. Neurosurgery 61:E1024-E1030, 2007

55. Tsuboi T, Watanabe H, Tanaka Y, Ohdake R, Yoneyama N, Hara K, et al: Distinct phenotypes of speech and voice disorders in Parkinson's disease after subthalamic nucleus deep 
brain stimulation. J Neurol Neurosurg Psychiatry 86:856864,2015

56. Tykocki T, Mandat T, Kornakiewicz A, Koziara H, Nauman P: Deep brain stimulation for refractory epilepsy. Arch Med Sci 8:805-816, 2012

57. Vertinsky AT, Coenen VA, Lang DJ, Kolind S, Honey CR, Li D, et al: Localization of the subthalamic nucleus: optimization with susceptibility-weighted phase MR imaging. AJNR Am J Neuroradiol 30:1717-1724, 2009

58. Volkmann J, Daniels C, Witt K: Neuropsychiatric effects of subthalamic neurostimulation in Parkinson disease. Nat Rev Neurol 6:487-498, 2010

59. Volkmann J, Fogel W, Krack P: Postoperatives neurologisches Management bei Stimulation des Nucleus subthalamicus. Akt Neurol 27 (Suppl 1):S23-S39, 2000

60. Wagle Shukla A, Okun MS: Surgical treatment of Parkinson's disease: patients, targets, devices, and approaches. Neurotherapeutics 11:47-59, 2014

61. Williams NR, Okun MS: Deep brain stimulation (DBS) at the interface of neurology and psychiatry. J Clin Invest 123:4546-4556, 2013

62. Witt K, Granert O, Daniels C, Volkmann J, Falk D, van Eimeren T, et al: Relation of lead trajectory and electrode position to neuropsychological outcomes of subthalamic neurostimulation in Parkinson's disease: results from a randomized trial. Brain 136:2109-2119, 2013

63. Wyman-Chick KA: Verbal fluency in Parkinson's patients with and without bilateral deep brain stimulation of the subthalamic nucleus: a meta-analysis. J Int Neuropsychol Soc 22:478-485, 2016

64. Yagmurlu K, Rhoton AL Jr, Tanriover N, Bennett JA: Threedimensional microsurgical anatomy and the safe entry zones of the brainstem. Neurosurgery 10 (Suppl 4):602-620, 2014

65. Yelnik J, Bardinet E, Dormont D, Malandain G, Ourselin S, Tandé D, et al: A three-dimensional, histological and deformable atlas of the human basal ganglia. I. Atlas construction based on immunohistochemical and MRI data. Neuroimage 34:618-638, 2007

66. Yoshida M: Creation of a three-dimensional atlas by interpolation from Schaltenbrand-Bailey's atlas. Appl Neurophysiol 50:45-48, 1987
67. Yoshida M: Three-dimensional electrophysiological atlas created by computer mapping of clinical responses elicited on stimulation of human subcortical structures. Stereotact Funct Neurosurg 60:127-134, 1993

68. Zemmoura I, Serres B, Andersson F, Barantin L, Tauber C, Filipiak I, et al: FIBRASCAN: a novel method for 3D white matter tract reconstruction in MR space from cadaveric dissection. Neuroimage 103:106-118, 2014

\section{Disclosures}

The authors report no conflict of interest concerning the materials or methods used in this study or the findings specified in this paper.

\section{Author Contributions}

Conception and design: Tanriover, Güngör, Baydın, Holanda, Middlebrooks. Acquisition of data: Tanriover, Güngör, Baydın, Holanda, Middlebrooks. Analysis and interpretation of data: Tanriover, Güngör, Baydın. Drafting the article: Tanriover, Güngör, Baydın, Holanda, Isler, Foote. Critically revising the article: Tanriover, Güngör, Baydın, Isler, Foote. Reviewed submitted version of manuscript: Güngör, Baydın, Holanda, Middlebrooks, Isler, Tugcu, Foote. Approved the final version of the manuscript on behalf of all authors: Tanriover. Administrative/technical/material support: Güngör. Study supervision: Tanriover, Foote.

\section{Supplemental Information}

Previous Presentations

Portions of this work were accepted in abstract form as proceedings at the World Federation of Neurosurgical Societies 16th World Congress of Neurosurgery, Istanbul, Turkey, August 20-25, 2017.

\section{Correspondence}

Necmettin Tanriover: Istanbul University, Istanbul, Turkey. nctan27@yahoo.com. 